\title{
RADIATION EQUILIBRIUM IN AN OPAQUE UNIFORMLY HEATED SOLID AND DUANE'S HYPOTHESIS AS TO ITS ORIGIN.
}

\author{
By A. G. Worthing.
}

WHETHER or not the natural radiation from the ultimate sources of radiation in an opaque uniformly heated solid agrees in spectral distribution with the equilibrium radiation in their immediate neighborhood depends on the optical constants of the solid. The condition for agreement is constancy in $\frac{k}{\lambda}$, where $k$ (often written $n k)$ is the extinction coefficient and $\lambda$ the wave-length in vacuo. A considerable tendency toward constancy exists in the measured values of this ratio. Possibly the variations found are real but analogous to those experienced in atomic heats in their relation to Dulong and Petit's law. Whatever the explanation, known difficulties due to surface transition layers seem to justify a tentative assumption of constancy for the purpose of testing theories of the origin of radiation.

Duane's hypothesis, ${ }^{1}$ a generalization from X-ray observations, ascribes all radiation to the bombardment of atoms by moving electrons-in the ordinary temperature radiations of incandescent solids the free electrons connected with electric conduction. Starting with three fundamental assumptions, Duane concluded that Planck's distribution law results. Apparently, however, he overlooked the fact that the coefficient $\mathrm{A}$ in his statement of Maxwell's law is in general a function of temperature. Fortunately, however, thermionic emission experiments seem to indicate that the concentration of the free electrons in a solid varies in such a fashion, at least for low concentrations and high temperatures, if not in general, as to render the coefficient A constant for this special case.

It is to be inferred from Duane's derivation that the natural radiation from the ultimate sources has the characteristics of black body radiation for free space. This is almost certainly not the case. Black radiation in free space differs from the equilibrium radiation in an opaque solid, and only in case $\frac{k}{\lambda}$ is constant, as stated above, has this equilibrium radiation the same spectral distribution as the natural radiation from the ultimate sources.

\footnotetext{
${ }^{1}$ Phys. Rev., 7, 143, 19г6.
} 
Assuming the constancy of $\frac{k}{\lambda}$ and thus for the natural radiation the same spectral distribution as for equilibrium radiation, it seems necessary to modify Duane's assumption (3) so as to read the energy radiated per hit is on the acerage proportional to the fourth power of the speed of the free electron and to the square of the index of refraction for the radiation emitted. In other words," the ratio of the energy radiated per impact to the kinetic energy of the impacting electron is proportional on the average to the square of the ratio of the velocity of propagation of the radiation in the solid to that in vacuo. On the new basis Planck's equation follows without question as to applicability.

In addition to giving cartain indications regarding the application of Maxwell's law to free electrons, and pointing out the possibility of a constancy in $\frac{k}{\lambda}$, the discussion shows how Duane's hypothesis, when combined with the assumption of such constancy and with conclusions arrived at independently in other lines of research, gives a satisfying physical concept of processes which may underlie the emission of radiation by its ultimate sources.

Nian Research Laboratory of Plre Science,

Neil Park, Cleveland, Ohio.

Positive Ray Analysis of Zinc. A. J. Dempster. (Science, Nov. 25, I92 I.) -From the Ryerson Physical Laboratory comes the announcement that zinc has been found to consist of four isotopes of respective atomic weights $-63,65,67$ and 69 . The relative intensities in the same order are 6:7:10: I From these atomic weights of the isotopes and the proportions in which they occur in ordinary zinc the atomic weight of the latter is calculated to be 65.5 . G. F. S.

On the Theory of Relativity and the Experiment of Sagnac. P. Langevin. (Comptes Rendus, Nov. 7, I92I.) - Sagnac caused two rays of light from the same source to interfere after they had traversed a closed circuit in opposite directions. When the entire optical system was set in rotation the interference fringes were shifted by an amount which corresponderl to an apparent difference between the times required to traverse the circuit in the two directions. Langevin derives Sagnac's exact expression for this time difference, founding his argument on the Theory of Relativity.

G. F. S.

As reworded by the writer's colleague, Dr. E. Q. Adams. 